http://jmscr.igmpublication.org/home/ ISSN (e)-2347-176x ISSN (p) 2455-0450 crossref DOI: https://dx.doi.org/10.18535/jmscr/v7i11.146

Journal Of Medical Science And Clinical Research

\title{
Ultrasound in Hashimoto's Thyroiditis in Young Female Presented as Hypothyroidism- A Case Report with Review
}

\author{
Authors

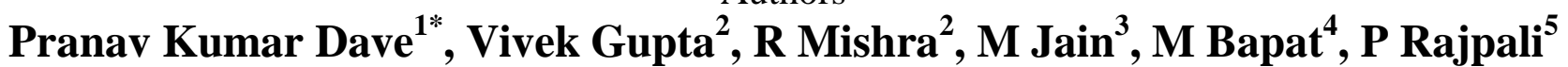 \\ M Tilgam5 , P Gupta ${ }^{5}$, A Patidar ${ }^{5}$, J Verma $^{6} \mathrm{C}_{\text {Dave }}^{7}$, P Saxena $^{8}$, V Agrwal $^{9}$
}

${ }^{1}$ Asso.Prof., ${ }^{2}$ Prof., ${ }^{3}$ Prof \& HOD, ${ }^{4}$ Asst. Prof., ${ }^{5}$ Resident, Department of Radio diagnosis, ${ }^{6}$ Prof of Pediatrics ${ }^{7}$ Ex.Asst. Prof,- Department of Pediatrics, ${ }^{8}$ Asst. Prof of Pathology, ${ }^{9}$ Prof. of Pathology L N Medical College and J K Hospital, Kolar Road, Bhopal-462042,MP, India.

*Corresponding Author

Dr Pranav K Dave

Asso. Prof. Deptt. of Radiology

\begin{abstract}
Thyroiditis is inflammatory disorder of thyroid with wide spectrum of clinical presentation. Thyroid function may be normal, high or low in valve. The presentation of symptoms, family history is of importance. Hashimoto's thyroiditis is not an uncommon cause of hypothyroidism in young population. Ultrasound is the imaging modality of choice in diagnosis of thyroid disorders. Thyroid ultrasonography is a simple, non -invasive, reproducible and without use of radiation with high sensitivity in diagnosis of thyroiditis. The purpose of this study was to determine the sensitivity and specificity of gray scale sonographic findings in Hashimoto's thyroiditis and its clinico-cytological correlation.

Introduction: Thyroiditis is defined as the inflammation of the thyroid gland and classified as acute/sub acute, chronic or autoimmune thyroiditis. Autoimmune thyroid disease AITD includes two major clinical presentations; Grave's Disease (GD) and Hashimoto's Thyroiditis $(H T)^{1}$. The prevalence of AITD is approximately 5\% with clinical presentation of hyperthyroidism or hypothroidism ${ }^{2}$. The pathogenesis is not very clear, there may be environmental factors, infection, drugs, smoking, etc. ,could trigger AITD in susceptible persons ${ }^{3}$.TUS findings in HT includes diffuse enlargement with decreased echogenicity, heterogeneity with hypervascularity and presence of hypo echoic micro nodules with echogenic rim. Cytological diagnosis is superior in children and as in initial stages of HT, antibody production may be limited. FNAC is quick, cost-effective and highly sensitive in diagnosis of HT.Most of the HT cases are in young and pre-menopausal females and more prone for poor obstetrical and fetal outcome, therefore early diagnosis of HT on clinical, TUS and cytology is of importance for supplementation of thyroxin for associated hypothyroidism.
\end{abstract}

Keywords: Hashimoto's thyroiditis HT, Grave's Disease GD, Autoimmune Thyroid disease AITD, Hypothyroidism, Thyroid Ultra Sonography-TUS, CD Color Doppler.

\section{Case Report}

A 13 year female child referred for TUS for evaluation of neck swelling .Parents noticed the swelling which was slowly increasing in size for last 2 years. Child has gained the weight during this duration. Her weight is $40 \mathrm{Kg}$, She gained about $7 \mathrm{~kg}$ weight in last 6 months duration 


\section{JMSCR Vol||07||Issue||11||Page 841-847||November}

She is fourth child and full term normal delivered in hospital. She is not from the endemic belt of iodine defiance area. Her Family history is non specific. Her siblings, two elder brothers and one elder sister were normal. On physical examination the child was anemic with swelling in lower neck region suggestive of thyroid origin. It was firm with uniform enlargement of thyroid gland. Her systemic examination was unremarkable. $\mathrm{Hb}$ was $-10 \mathrm{gm} / \mathrm{dl}$. Skiagram chest reveals no abnormality. TUS findings reveals diffuse enlargement of both lobes and isthmus of thyroid. Heterogenous echo texture intercepted with multiple ill-defined hypo echoic areas seen in both lobes and in isthmus. On $\mathrm{CD}$ there no abnormal blood flows. Few enlarged hypo echoic cervical lymph nodes seen on either side, suggestive of reactive lymph nodes. Ultrasound of abdomen and pelvis was normal.

Her thyroid hormonal profile readings were T3$191 \mathrm{ng} / \mathrm{dl}$. T4 -7.94mg/dl and TSH.-15.3 mIU/ml. suggestive of hypothyroidism. And High Anti thyrioglobulin antibody-296I U/ml. suggestive of thyroiditis. Other Para meters including her $\mathrm{Hb}$ were within normal limits.

Under aseptic precaution US guided FNAC was performed, which revealed -Hurtle cell chine with anisonucleosis and varying amount of polymorphous lymphoid infiltrate in a background of scant colloid.

The exact cause of HT could not be established in this case, High possibility of iatrogenic in origin was considered.

After establishment of HT, she was recommended hormonal supplementation therapy and advice for regular follow up.

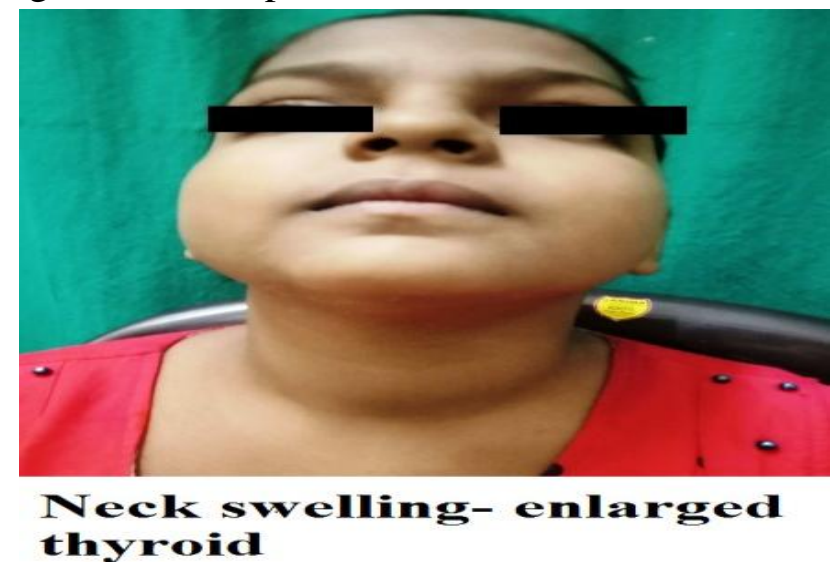

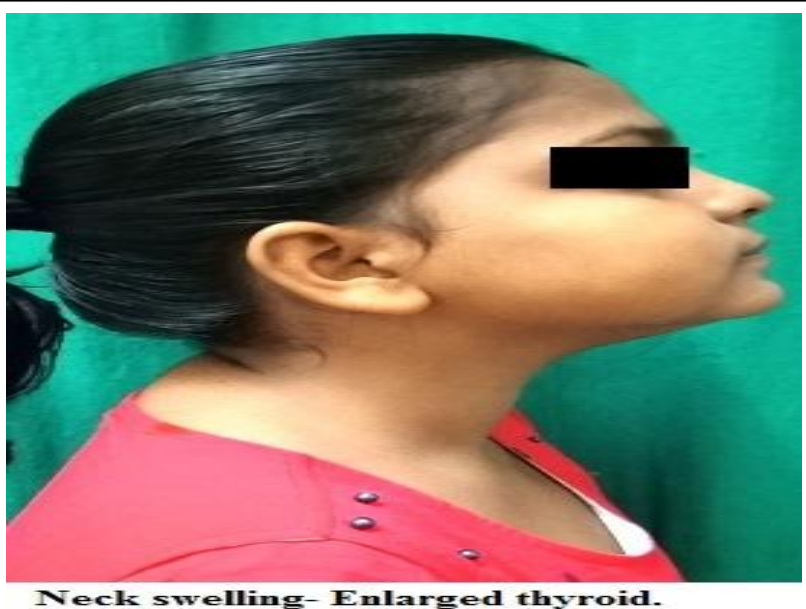

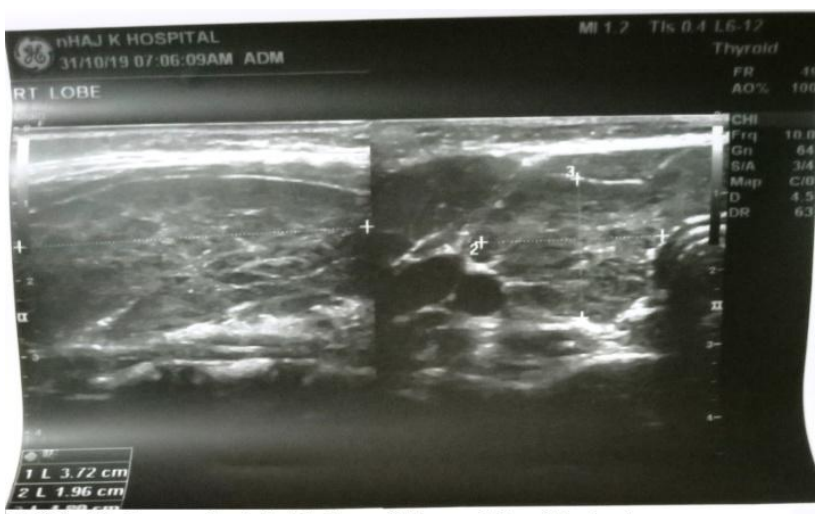

TUS- Enlarged right lobe of thyroid with heterogenous echotexture.

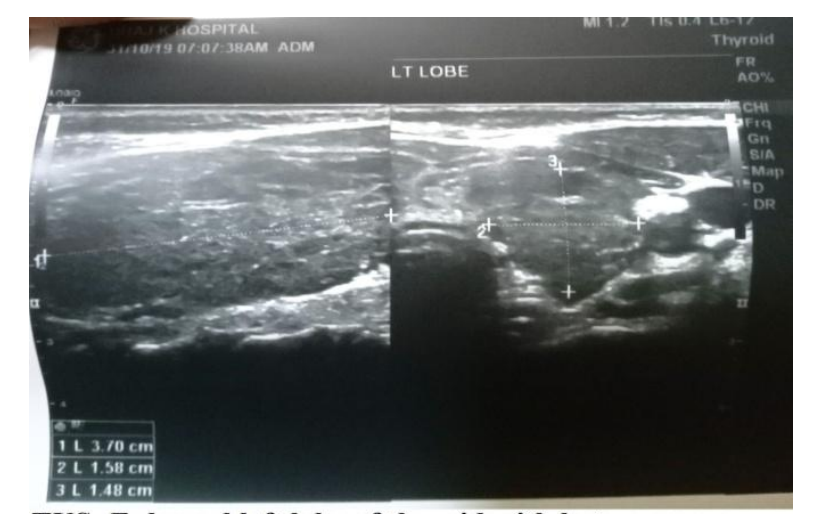

TUS- Enlarged left lobe of thyroid with heterogenous echotexture.

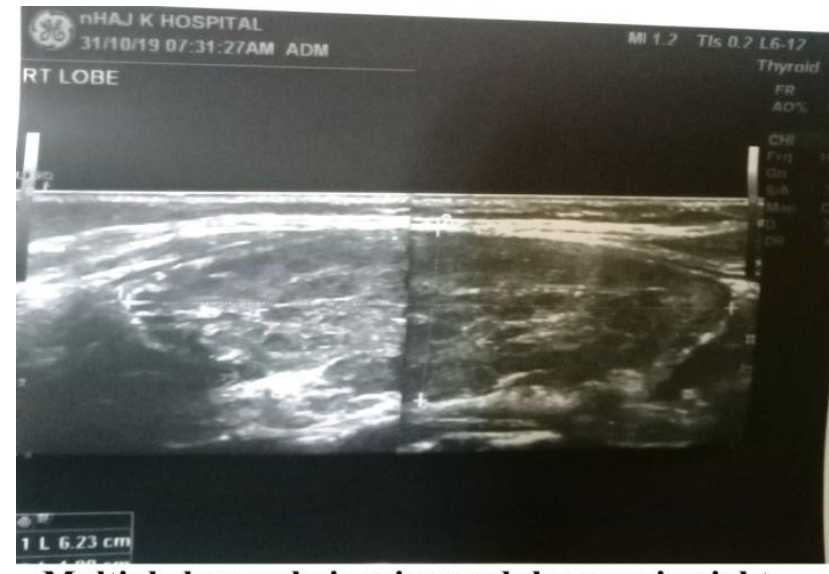

Multiple hypoechoic micronodules seen in right lobe of thyroid. 


\section{JMSCR Vol||07||Issue\|11||Page 841-847||November}

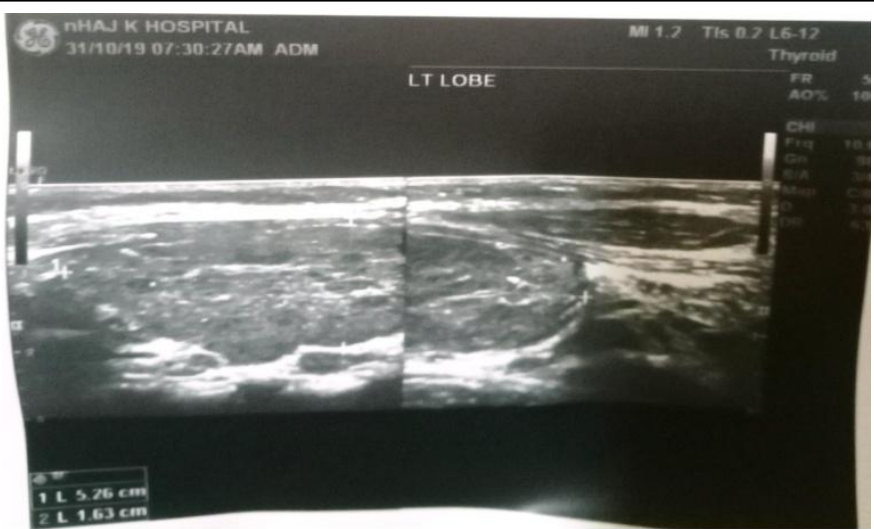

Hypoechoic micronodules seen in left lobe of thyroid.

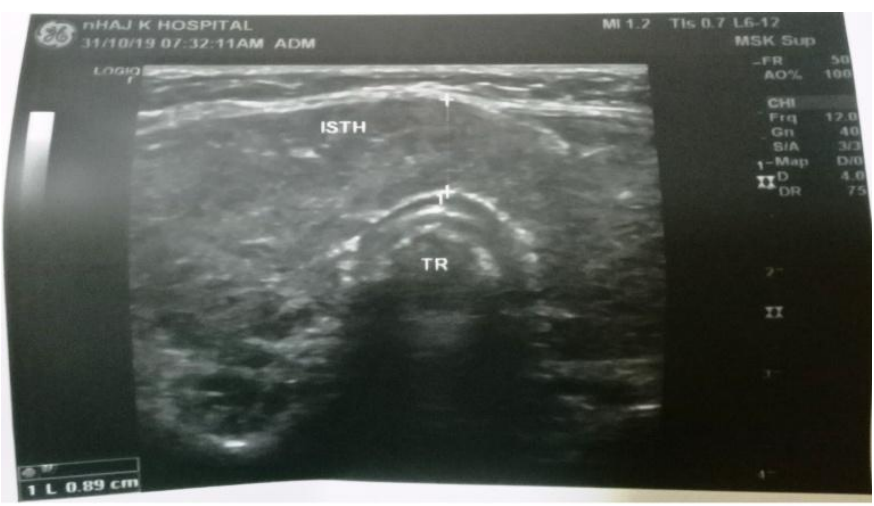

Enlarged isthmus of thyroid.

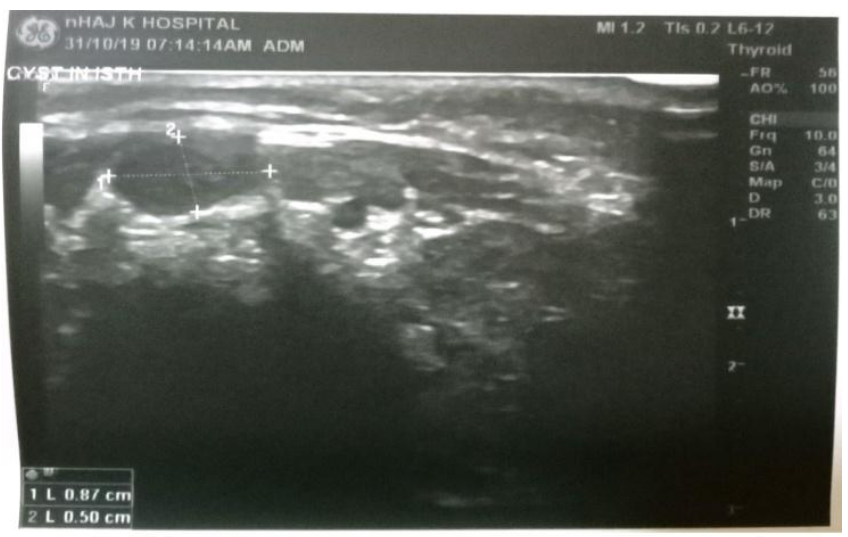

Cystic degeneration seen in isthmus of thyroid.

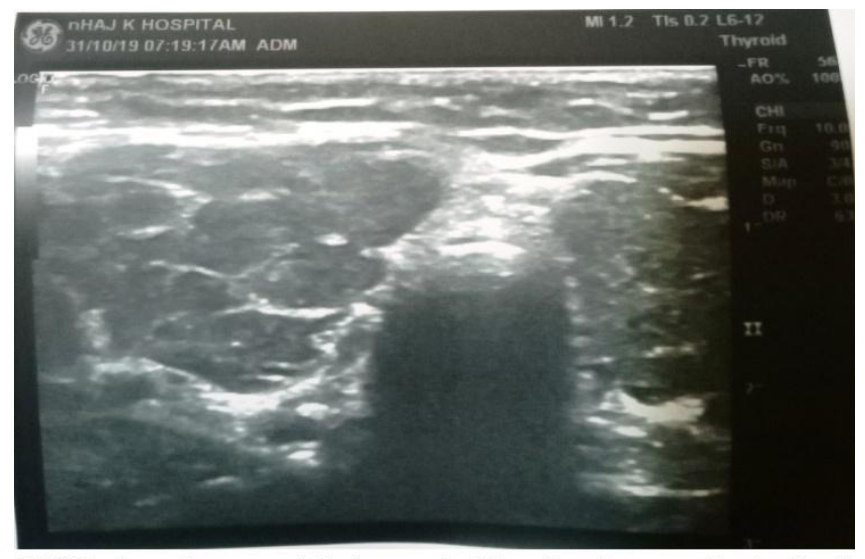

TUS showing multiple variable size hypoechoic foci.

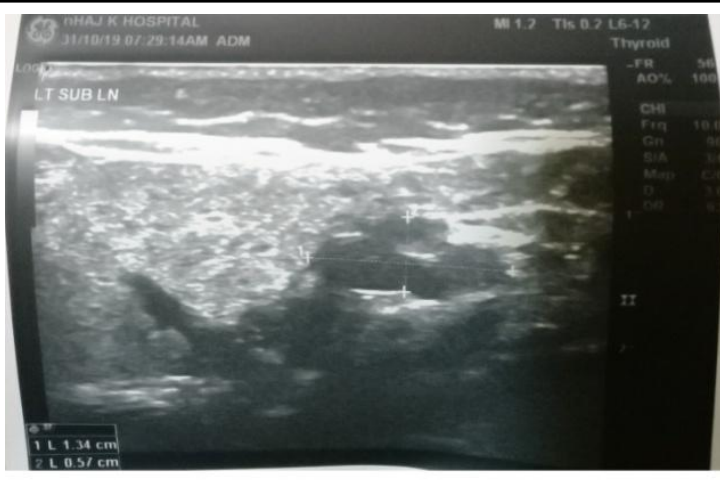

Hypoechoic enlarged cervical lymph node.

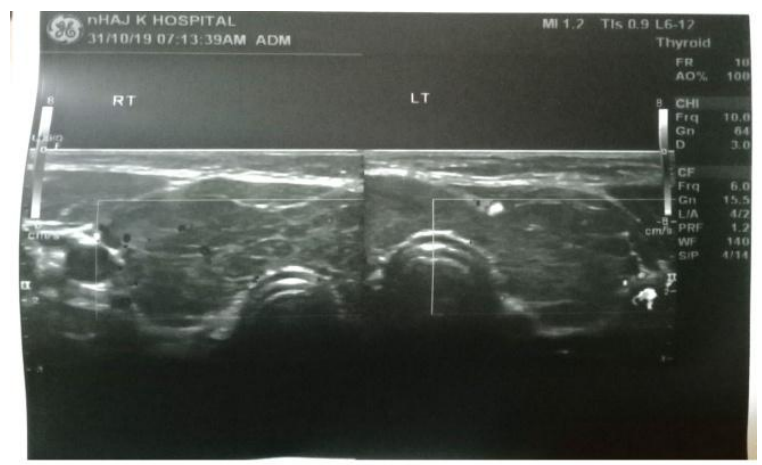

COLOR DOPPLER- showing hypovascularity of both lobes of thyroid.

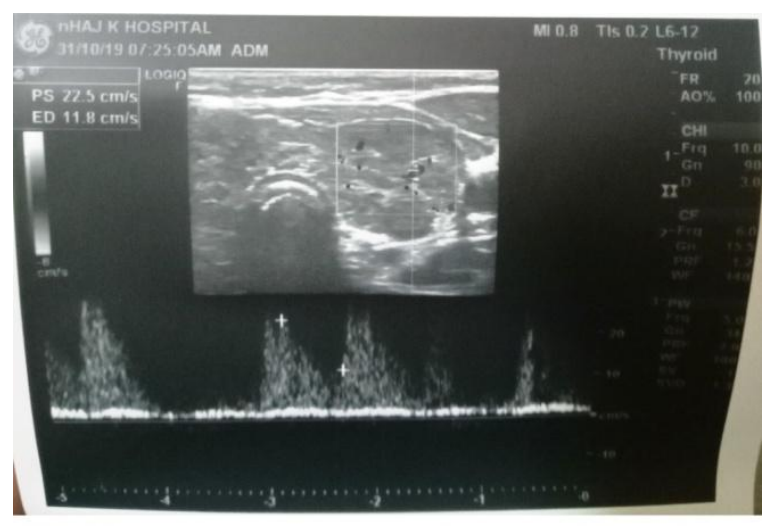

COLOR DOPPLER- Hypovascularity in left lobe of thyroid.

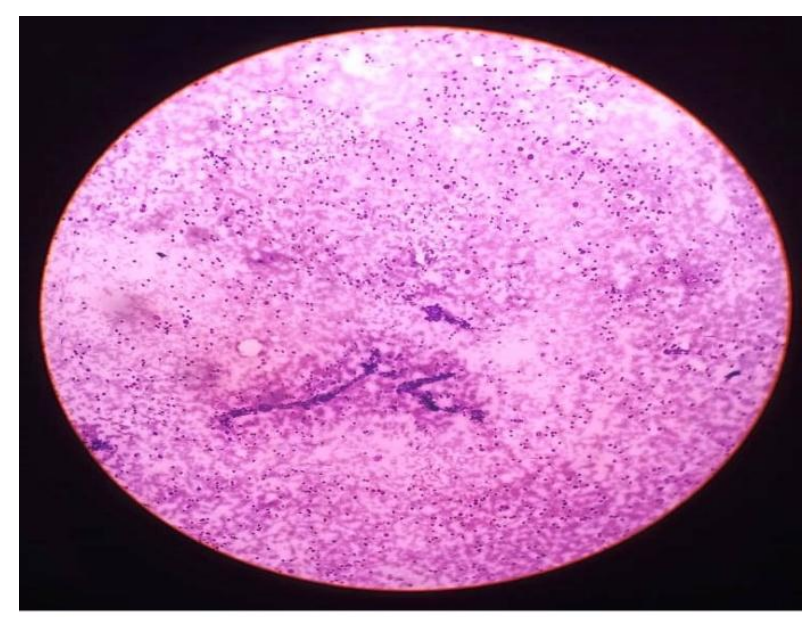

FNAC-Clusters of benign thyroid follicular cells with impingement of lymphocytes and scanty colloid. 


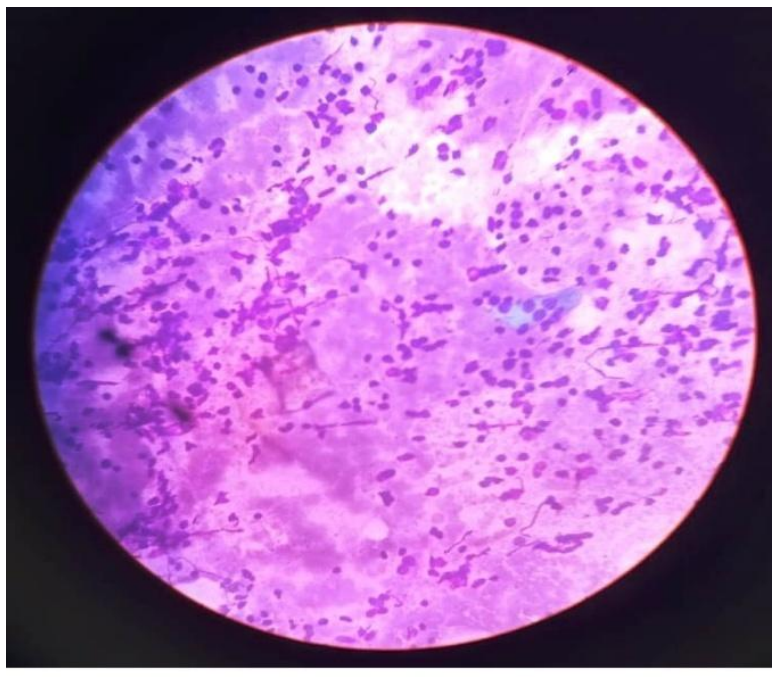

FNAC-Clusters of benign thyroid follicular cells with impingement of lymphocytes and scanty colloid.

\section{Discussion}

The autoimmune thyroiditis represent a wide variety of disorder with a common finding of lymphocytic infiltration in thyroid parenchyma and production of anti thyroid antibodies ${ }^{5}$. Autoimmune Thyroid Disease constitute 30\% of all auto immune disorders and qualified as organ specific out of which Hashimoto's thyroiditis HT/ Chronic Lymphocytic Thyroiditis and Grave's disease are the most important ${ }^{6}$.HT was first described in 1912 by Hakaru Hashimoto, is the most common auto immune thyroid disease and the commonest cause of hypothyroidism ${ }^{7}$.He reported the findings in excised thyroid gland specimens from four women who presented with unusual type of goiter with the peculiar clinical and histological findings which combined a non specific or even hypothyroidism symptoms with a diffuse and massive lymphatic infiltration in the gland. This report resulted in better understanding the most common thyroid disease and HT,as in many cases used interchangeably with the term chronic lymphocytic / autoimmune thyroiditis. Chronic autoimmune lymphocytic thyroiditis represent as a most common cause of hypothyroidism and the most frequent autoimmune disease affecting human beings ${ }^{5}$. In HT there is lymphocytic infiltration of the thyroid gland followed by a gradual destruction and fibrous replacement of the thyroid parenchyma ${ }^{8}$.
Individual may present with goiter. HT occurs in all ages but most commonly seen between 30-50 years, but usually manifest after 50 years of age. It affects $5 \%-15 \%$ of female and $1 \%-5 \%$ of male, almost nine times more frequent in female according to diagnostic criteria and geographic location ${ }^{5}$. HT may be associated with other autoimmune disease such as lupus, Grave's disease or pernicious anaemia ${ }^{5}$. With advancement Ultrasound technology is also useful evaluation of various thyroid pathologies. Several parameters, like glandular volume, echo texture, echogenicity and vascular status are evaluated in TUS ultrasound which contribute to the diagnosis of thyroiditis ${ }^{5-9}$.

The echogenicity of thyroid parenchyma is compared with the echogenicity of the pre-thyroid muscles and the sub mandibular gland and differentiated as isoechogenic, hyperechogenic and hypoechogenic. Normal thyroid gland is more echogenic as compared to the pre thyroid muscles and slightly higher than the submandibular gland. The hypo echoic thyroid parenchyma is considered when its echo levels are similar or lower than the submandibular glands but higher than muscles ${ }^{5}$. There is high concentration of serum thyroid antibodies in $\mathrm{HT}^{5}$. The HT may be in nodular focal form or diffuse form ${ }^{10}$. Nodular focal form seen as a small size focal hypo echoic thyroid nodule with ill-defined margins .Color Doppler findings are non specific in these nodules. The diffuse form usually present as an enlarged thyroid gland with multiple small hypo echoic nodules on ultrasound study ${ }^{11}$. This is due to focal lymphocyte infiltration in between normal areas of thyroid parenchyma and fibrosis. This pattern resembles a giraffe hide pattern ${ }^{12}$.Thryoid gland diffusely enlarged with progress of disease into chronic hypertrophic thyroiditis results in pseudo lobulated hypoechoic and with multiple hypo echoic pseudo nodules separated by fibrotic bands on TUS. Advance cases results into atrophic form in which the thyroid gland becomes small in size with ill-defined contours and diffusely heterogenous parenchyma. Enlarged 
reactive cervical nodes are seen in some cases. In some cases uniformly hyper echoic ("White knight") pattern seen due to interspersed with hypo echoic areas of lymphocytic infiltration seen on TUS. These areas do not present peculiar vascularization on color doppler study. These pseudo nodular areas should not be confused with true nodules as seen in multi nodular goitre $^{5}$.Diffuse hyper vascularization as "thyroid inferno" similar to Graves' disease is seen early stage on $\mathrm{CD}$.This is due to less intense form with lower systolic velocity peak in thyroid arteries. In later stages of HT, CD findings are usually of diffusely hypo vascularization and sometimes even no detectable blood flow ${ }^{13}$. Ill-defined margins small size gland with diffusely heterogenous parenchyma and no flow on $\mathrm{CD}$ seen in late chronic phase of HT. A rapid growing nodule should raise the suspicion of a primary thyroid lymphoma, because this is 60 to 80 times more in known cases of HT. Papillary carcinoma may also be associated with $\mathrm{HT}^{14}$. In some cases HT may coexist with Graves's disease and in these cases CD will demonstrate an increase in thyroid blood flow with systolic velocity peak in the thyroid arteries over $40 \mathrm{~cm} / \mathrm{s}^{15}$.

TUS findings of HT include diffuse enlargement of gland with decreased echogenicity, heretogeneity, hypervascularity and presence of hypo echoic micro nodules with echogenic rims $^{7,16}$. Decreased blood flow was observed on $\mathrm{CD}$ in few study as differentiating feature from Grave's disease $^{7,17}$. Usual presentation of HT is as firm and painless variable size of enlargement of thyroid gland. Rarely it is painful and often ovulated appearance makes difficult to differentiate with multinodular goiter .Pain, rapid enlargement in size with enhanced posterior echoes on TUS may indicate primary thyroid Bcell lymphoma $^{7-18}$.

Prolonged stimulation of intrathyroidal B cells results in emergence of malignant clone. A FNAC/B with immunophenotypic analysis may be indicated if there is pain with rapid enlargement of goitre. There is controversy for the link between HT and papillary thyroid carcinoma (PTC). At present there is no valid established criterion to identify those cases with $\mathrm{HT}$ at a higher risk of developing PTC.A regular follow up of HT cases is recommended, particularly in nodular variants. Thomas et $\mathrm{al}^{7}, 18$ studied the clinical, biochemical, antibody levels, ultrasound and cytomorphologic characteristics of 144 cases with cytological diagnosis of HT in an endemic zone for goiter with a widespread use of iodized salt and found that 90 percent of HT cases were females mainly between 21-40 years of age similar to other Indian studies ${ }^{7}$. In western literature HT occurs seven times more in females with a peak incidence in between 40-60 years of age $^{7}$ with symmetrical, firm and diffuse painless goiter in 90 percent of HT cases. Thomas et $\mathrm{al}^{19}$ reported that goiter was the commonest presenting complaint with majority (61\%) having diffuse goiter. Most (46\%) were hypothyroid (subclinical and overt), $33 \%$ cases were euthyroid and $21 \%$ were hyperthyroid on evaluation. Among the hypothyroid cases, only $38 \%$ showed overt hypothyroidism similar to previous studies in India $^{7,20 .}$ HT may coexist with Graves's disease. Thomas et $\mathrm{al}^{19}$ found that $93 \%$ of the study subjects with HT were antiTPO positive and 92\% were antithyroglobulin antibodies (Anti $\mathrm{Tg}$ ) positive. Pearce et $\mathrm{al}^{7}$ found TPO antibodies in $90-95 \%$ of HT but anti - Tg antibodies in only 20$50 \%$.TSH receptor blocking antibodies may cause transient hypothyroidism in infants born to the mother with $\mathrm{HT}^{7}$. TUS in current study showed multiple micro hyopechoic areas in enlarged thyroid with heterogenous echo texture and decreased vascularity. With HT, Primary carcinoma thyroid and primary thyroid lymphoma are common associated neoplasm. Primary thyroid lymphoma is 60 to 80 times more common in cases with HT than the general population ${ }^{7}$ .Thomas et al ${ }^{19}$ suggested screening of all women of reproductive age for HT in an endemic zone for goiter's D.Sanyal ${ }^{7}$ reported that the importance of iodine deficiency and endemic goiter, thyroid autonomy, non- autoimmune nodular 
hyperthyroidism and congenital hypothyroidism cannot be underestimated in the Indian context. Iodine deficiency decreased prevalence of goiter and congenital hypothyroidism in young population and reduced frequency of nonautoimmune autonomous hyperthyroidism in older age group far outweighs the risk of development of thyroid autoimmunity and mild hypothyroidism in youngster ${ }^{7}$. HT is the commonest cause of goiter and hypothyroidism especially in females of reproductive age group in the post iodization era. Euthyroid stage of HT may progress to overt hypothyroidism in young age group. Goiter or Clinical hypothyroidism are indications for the treatment and follow up of HT cases. Future research is needed for the early diagnosis of HT. Early diagnosis of unrecognized hypothyroidism in young and pregnant female may avoid future complications. Present case child was diagnosed as HT with hypothyroidism, supplementation of hormone was given and advice for regular follow.

\section{Conclusion}

Our findings indicate that sonography has high specificity with good sensitivity in the diagnosis of Hashimoto's thyroiditis. Regular TUS monitoring is important in case of HT and to differentiate other thyroid diseases. However confirmation of HT by laboratory data and cytology is needed.

Financial support and sponsorship - Nil

Conflicts of Interest -There are no conflicts of interest.

\section{References}

1. Zhaohui Cui.et al. Establishment of clinical diagnosis model of Grave's disease and Hashimoto' thyroiditis. Journal of Translational Medicine. 2019,17:11, http://doi.org/10.1186/s12967-018-1765-3.

2. Antonell A et al Autoimmune thyroid disorders. Autoimmune. Rev. 2015;14(2):174-80
3. Shukla SK. etal. Infections, genetic and environmental factors in pathogenesis of autoimmune thyroid disease .Mico Pathog. 2018;116:279-88.

4. Shetty A. Chowdappa V. Cytomorphological Spectrum of Hashimoto;s Thyroiditis and Its Correlation with Hormonal Profile and Hematological Parameters. Journal of Cytology.2019;36:137-41.

5. Takahashi MS et al. Ultrasound Evaluation of Thyroiditis: A Review.Journal of Otolaryngology

Research.2019;2(1):127.01-09.

6. Aleksandra Pyzik et al. Immune Disorders in Hashimoto's Thyroiditis: What Do We Know So Far ?. Journal of Immunology Research. Volume 2015,Article ID 979167,8 pages.

7. Debmalya Sanyal. Spectrum of Hashimoto's Thyroidits: Clinical, Biocchemical \& Cytomorphologic profile. Indian J Med Res.140, December 2014, pp.710-712.

8. Katja Zalete and Simona Gaberscek. Hashimoto's Thyroiditis: From Genes to the Disease. Current Genomics, 2011, 12,576-588.

9. Mazziotti G. Gray-scale analysis allows a quantitative evaluation of thyroid echogenicity in the patients with Hashimoto's thyroiditis. Clin Endocrinol (Oxf). 2003.59:223-229.

10. Langer JE et al. Sonographic apperance of focal thyroiditis. AJR. Am J Roentgenol. 2001.176:751-754.

11. Yeh $\mathrm{HC}$ et al. Micronodulation: Ultrasonographic sign of Hahimoto;s thyroiditis.J Ultrasound Med .1996.15:813-819.

12. Virmani V, Hammond I.Sonographic patterns of benign thyroid nodules: verification at our institution .AJR,Am J Roentgenol.2011,196:891-895. 
13. Sawin CT. Hakaru Hashimoto and his disease The Endocrinologist 2002.49: 399403.

14. Halm LE. Cancer risks in patients with chronic lymphocytic thyroiditis. N Engl.J Med 1985.312:601-604.

15. Dos Santos TARR et al. Grave's Disease Thyroid Color-Flow Doppler Ultrasonography Assessment Health 2014,6:1487-1496.

16. Anderson L et al.Hashimoto thyroiditis.Part2. Sonographic analysis of benign and malignant nodules in patients with diffuse Hashimoto thyroiditis. AJR, Am J Roentgenol.2010.195:216-22.

17. Donkol R H et al.Role of color Doppler in differentiation of Graves' disease and thyroiditis in thyrotoxicosis. World $\mathbf{J}$ Radiol.2013;5:178-83.

18. Stein S A et al.primary thyroid lymphoma: a clinical review. J Clin Endocrinol. Metlab.2013;89:3131-8.

19. Thomas $\mathrm{T}$ et al. Clinical, biochemical \& Cytomorphphologic study on Hashimoto's thyroiditis. Indian J Med. Res. 2014;140:729-35.

20. Bhatia A .et al. Lymphocytic thyroiditis- is cytological grading significant?.A correlation of grades with clinical, biochemical, ultrasonographic and radionuclide parameters. Cytojournal. 2007:4:10. 